\title{
Fatores de risco para mortalidade hospitalar nas reoperações valvares
}

\author{
Carlos Manuel de Almeida BRANDÃO, Pablo Maria Alberto POMERANTZEFF, Luciano Rapold SOUZA, Flávio \\ TARASOUTCHI, Max GRIMBERG, Sérgio Almeida de OLIVEIRA
}

RBCCV 44205-595

\section{Resumo}

Objetivo: Identificar fatores de risco para mortalidade hospitalar em reoperações valvares.

Método: Foi realizada análise prospectiva de 194 pacientes submetidos a reoperações valvares no período entre julho de 1995 e junho de 1999. As variáveis estudadas foram: sexo, idade, classe funcional, número e tipo de operações prévias, intervalo entre as operações, caráter da operação, creatinina sérica, fração de ejeção do ventrículo esquerdo, diâmetros diastólico e sistólico do ventrículo esquerdo, pressão sistólica de ventrículo direito, atividade de protrombina, relação do tempo de tromboplastina parcial ativada, contagem de plaquetas, tempo de circulação extracorpórea, tempo de pinçamento aórtico, posição e número de valvas, tipo de procedimento, operações associadas e volume de sangramento intra-operatório. Análise univariada e multivariada foi realizada para determinar os fatores de risco para mortalidade hospitalar.

Resultados: A mortalidade hospitalar foi de 8,8\% (17 pacientes). A análise univariada identificou as seguintes variáveis associadas a maior mortalidade: classe funcional avançada, fração de ejeção do ventrículo esquerdo baixa, atividade de protrombina baixa, creatinina elevada, tempo de circulação extracorpórea prolongado, tempo de pinçamento aórtico prolongado, procedimentos associados e volume de sangramento intra- operatório elevado. Na análise multivariada foram significativas: classe funcional IV, creatinina $>1,5 \mathrm{mg} / \mathrm{dl}$ e tempo de circulação extracorpórea > 120 minutos.

Conclusões: As variáveis classe funcional IV, creatinina > $1,5 \mathrm{mg} / \mathrm{dl}$ e tempo de circulação extracorpórea $>120$ minutos são fatores de risco independentes para mortalidade hospitalar nas reoperações valvares.

Descritores: Valvas cardíacas, cirurgia. Fatores de risco.

\begin{abstract}
Objective: Analyse the risk factors for hospital mortality in valvar reoperations.

Method: A prospective analysis was performed of 194 patients that underwent valvar reoperations between July 1995 and June 1999. The following variables were analysed: sex, age, functional class, number and type of previous operations, cardiac rhythm, urgency at operation, creatinin level, left ventricular ejection fraction, left ventricular systolic and diastolic diameters, right ventricular systolic pressure, prothrombin activity, activated partial thromboplastin time relation, platelets count, extracorporeal circulation time, aortic cross-clamp time, number and position of valves, type of procedure, associated procedures and intraoperative bleeding volume. Univariate and multivariate statistical analysis was performed to determine the risk factors for hospital mortality.

Results: The overall hospital mortality was $8,8 \%(17$ patients). Univariate analysis showed that the following variables were associated with higher mortality rates: advanced New York Heart Association functional class, decreased left ventricular ejection fraction, decreased prothrombin activity, increased creatinine level, longer aortic cross-clamp time, prolonged extracorporeal circulation time, concomitant associated procedures, and higher intraoperative bleeding volume. Logistic multivariate analysis identified advanced New York Heart Association functional class, creatinine level higher than $1,5 \mathrm{mg} / \mathrm{dl}$, and extracorporeal circulation time longer than 120 minutes as independent predictors of hospital mortality.

Conclusions: The variables functional class IV, creatinin level $>1,5 \mathrm{mg} / \mathrm{dl}$ and extracorporeal circulation time $>120$ min were independent predictors of hospital mortality in valvar reoperations.
\end{abstract}

Descriptors: Heart valves, surgery. Risk factors.

Trabalho realizado no Instituto do Coração do Hospital das Clínicas da Faculdade de Medicina da Universidade de São Paulo. São Paulo, SP, Brasil.

Apresentado ao $28^{\circ}$ Congresso Nacional de Cirurgia Cardíaca. Ouro Preto, MG, 5 a 7 de abril de 2001.

*Do Instituto do Coração do Hospital das Clínicas da Faculdade de Medicina da Universidade de São Paulo.

Endereço para correspondência: Carlos Manuel de Almeida

Brandão. Av. Dr. Enéas de Carvalho Aguiar, 44. Cerqueira César.

São Paulo, SP, Brasil. CEP: 05403-000. Tel: (11) 30695638.

Fax: (11) 30695415. E-mail:carlos.brandao@incor.usp.br 


\section{INTRODUÇÃO}

Os pacientes submetidos a reoperações valvares constituem um grupo bastante heterogêneo, uma vez que incluem pacientes com disfunção estrutural de biopróteses ou re-estenoses valvares, endocardites em prótese, escape para-valvar ou trombose de prótese mecânica. Estes pacientes apresentam-se com uma variedade de condições clínicas, podendo ser assintomáticos, oligossintomáticos ou até mesmo estarem em choque cardiogênico. A intervenção cirúrgica nas reoperações exige acurácia técnica e cuidados especiais, essenciais para a obtenção de bons resultados.

Em nosso meio, o número de reoperações valvares é grande, devido principalmente à ampla utilização de biopróteses, que têm vida útil limitada pela disfunção estrutural. No período compreendido entre 1980 e 1999, no Instituto do Coração, dentre 7544 operações valvares foram implantadas 5502 próteses, sendo 4535 biopróteses, correspondendo a 82,4\%. Destas operações, $22,8 \%$ foram reoperações.

$\mathrm{Na}$ literatura internacional, várias análises univariadas ${ }^{(1,2)} \mathrm{e}$ multivariadas ${ }^{(3,4)}$ foram realizadas, identificando fatores de risco para morbidade e mortalidade hospitalar. Poucos trabalhos em nosso meio ${ }^{(5,6)}$ analisaram os fatores de risco nas reoperações valvares, não havendo nenhuma análise multivariada destes fatores, não ficando claro quais os fatores de risco independentes para mortalidade na nossa população.

O objetivo deste trabalho é identificar fatores de risco para a mortalidade hospitalar em pacientes submetidos a reoperações valvares no Instituto do Coração da Faculdade de Medicina da Universidade de São Paulo.

\section{MÉTODO}

Foi realizada análise prospectiva de 194 pacientes consecutivos submetidos a reoperações valvares no período compreendido entre julho de 1995 e junho de 1999. Foram incluídos neste estudo somente pacientes com antecedentes de operação valvar.

A indicação da reoperação foi disfunção estrutural de bioprótese em 129 (66,5\%) pacientes, sendo calcificação em 59e rotura em 70; disfunção de prótese mecânica em 9 (4,6\%) pacientes; das quais trombose de prótese em 1 e escape paravalvar em 8; endocardite em prótese em 13 (6,7\%) pacientes; endocardite em valva nativa em $1(0,5 \%)$ paciente, re-estenose em valva nativa em $32(16,5 \%)$ pacientes e insuficiência mitral pós-plástica em $10(5,1 \%)$ pacientes. A etiologia da lesão valvar primária foi febre reumática em $146(75,3 \%)$ pacientes, degenerativa em $20(10,3 \%)$, endocardite em $14(7,2 \%)$, congênita em $12(6,2 \%)$, cardiomiopatia dilatada em $1(0,5 \%)$ e traumática em $1(0,5 \%)$.

O intervalo entre as operações variou entre 1 mês e 43 anos, com média de $8,1+/-6,2$ anos. Foram realizadas substituições valvares em 178 (91,7\%) pacientes, com utilização de biopróteses em $144(74,2 \%)$ e próteses mecânicas em $34(17,5 \%)$. Dezesseis $(8,2 \%)$ pacientes foram submetidos a procedimentos conservadores.

Setenta e sete $(36,7 \%)$ pacientes, com idade superior a 40 anos ou com fatores de risco para doença coronariana foram submetidos a cineangiocoronariografia, sendo identificadas lesões coronárias críticas em $3(1,5 \%)$ pacientes. Estes foram submetidos a revascularização do miocárdio com enxertos de veia safena, todos coincidentemente para a artéria coronária direita. Em 6 (3,1\%) pacientes com endocardite encontramos abscesso de anel valvar aórtico, que foram tratados através de sutura direta em $5(2,6 \%)$ pacientes e através de reconstrução com retalho de pericárdio bovino em $1(0,5 \%)$ paciente. Em 1 $(0,5 \%)$ paciente, encontramos abscesso de anel valvar mitral, sendo tratado através de sutura direta.

Quanto à tática operatória, a via de acesso utilizada foi a esternotomia mediana, realizada com serra oscilante circular. Para minimizar a área de descolamento, a pleura esquerda foi sistematicamente aberta, permitindo uma adequada mobilização do coração. Prosseguíamos com a dissecção do átrio direito e da aorta. A circulação extracorpórea foi estabelecida com a canulação da aorta ascendente e das veias cavas superior e inferior. A proteção miocárdica foi realizada pela hipotermia moderada a $28 \mathrm{o}$, associada à hipotermia tópica e à solução cardioplégica cristalóide do tipo St Thomas, a cada 25 minutos, via anterógrada. Nos pacientes que já tinham próteses, iniciamos a retirada destas pela remoção dos fios de sutura prévios, o que permite a tração destas próteses com pinças do tipo Moyniham e o seu descolamento progressivo do anel valvar. Após a retirada da prótese, o excesso de fibrose ou restos de tecido dos implantes prévios foram sistematicamente retirados do anel valvar.

\section{Variáveis Pré-Operatórias}

Foram analisadas as seguintes variáveis clínicas: sexo, idade, classe funcional (CF), número de operações prévias, tipo de operação prévia, ritmo cardíaco e caráter da operação.

Cento e vinte e quatro $(63,9 \%)$ pacientes eram do sexo feminino e 70 (36,1\%) do masculino. Aidade variou entre 6 e 83 anos, com média de 47,4 anos e desvio-padrão de 17,3 anos. Para efeito da análise de risco, dividimos as faixas etárias em: menor ou igual a 16 anos (15 pacientes), entre 17 e 65 anos (144 pacientes) e maior do que 65 anos (35 pacientes). Quanto a CF, 20 (10,3\%) pacientes estavam em CF II, $104(53,6 \%)$ em CF III e $70(36,1 \%)$ emCFIV.

. O número médio de operações valvares prévias foi de $1,4+1$ $-0,7$ operações; sendo que $127(65,5 \%)$ pacientes tinham 1,52 (26,8\%) tinham 2, $13(6,7 \%)$ tinham $3,1(0,5 \%)$ tinha 4 e $1(0,5 \%)$ tinha 5 operações valvares prévias. Segundo o tipo de operação valvar prévia, $43(22,2 \%)$ pacientes foram submetidos a cirurgias conservadoras e $151(77,8 \%)$ a substituições valvares. Quanto ao ritmo cardíaco, 126 pacientes $(64,9 \%)$ apresentavam ritmo sinusal, 67 (34,5\%) apresentavam fibrilação atrial e $1(0,5 \%)$ apresentava bloqueio atrioventricular total com marcapasso atrioventricular universal. Cinco $(2,6 \%)$ operações foram realizadas em caráter de emergência, todas as demais foram eletivas. A indicação de emergência foi edema agudo pulmonar em 4 pacientes e choque séptico em 1. 
Foram analisados os seguintes parâmetros ecoDopplercardiográficos: fração de ejeção do ventrículo esquerdo (FEVE), diâmetro diastólico do ventrículo esquerdo (DDVE), diâmetro sistólico do ventrículo esquerdo (DSVE) e pressão sistólica do ventrículo direito (PSVD), que foram estratificadas de acordo com os valores normais ou alterados. A FEVE variou de 29 a $85 \%$, com média de $68,4+/-9,3 \%$. ODDVE variou de 3,4 a $10,7 \mathrm{~cm}$, com média de $5,4+/-0,9 \mathrm{~cm}$. O DSVE variou entre 2,1 a $8,5 \mathrm{~cm}$, com média de $3,6+/-0,8 \mathrm{~cm}$. APSVD variou entre 20 e $120 \mathrm{mmHg}$, com média de $56,9+/-19,9 \mathrm{mmHg}$.

Foram analisadas as seguintes variáveis laboratoriais: nível sérico de creatinina, atividade de protrombina (AP), relação do tempo de tromboplastina parcial ativada (rTTPA) e contagem de plaquetas, que foram divididas de acordo com os valores considerados normais ou alterados, de acordo com os métodos de dosagem utilizados. Acreatinina variou entre $0,4 \mathrm{e} 2,3 \mathrm{mg} / \mathrm{dl}$, com média de 1,0+/- 0,3 mg/dl. AAPvariou de 24,4 a 100\%, com média de $77,2+/-16,7 \%$. ArTTPA variou entre 0,6 e 2,4, com média de $1,1+/-0,2$. A contagem de plaquetas variou entre 67.000 e 380.000/mm3, com média de 194.902+/-63.459/mm3.

\section{Variáveis Intra-Operatórias}

Foram analisadas as seguintes variáveis intra-operatórias: o tempo de circulação extracorpórea (CEC), o tempo de pinçamento aórtico, o número de valvas, o tipo de procedimento, a posição, as operações associadas e o volume de sangramento intraoperatório.

O tempo de CEC variou entre 34 e 247 minutos, com média de $110,6+/-34,6$ minutos. Os pacientes foram estratificados em dois grupos: com tempo de CEC menor ou igual que 120 minutos (134 pacientes) e maior que 120 minutos (60 pacientes). Com relação ao tempo de pinçamento aórtico, que variou entre $22 \mathrm{e} 214$ minutos (média 91,2+/-31,3 minutos), os pacientes foram divididos em: menor ou igual a 90 minutos (115 pacientes) e acima de 90 minutos (79 pacientes).

O volume de sangramento intra-operatório variou entre 1,1 e $52,0 \mathrm{ml} / \mathrm{Kg}$ de peso. Para a análise de risco, dividimos os pacientes em dois grupos, segundo o valor médio de sangramento $(8,6 \mathrm{ml} /$ $\mathrm{kg}$ ): menor ou igual a $8,6 \mathrm{ml} / \mathrm{kg}$ (120 pacientes) e maior que $8,6 \mathrm{ml} /$ $\operatorname{kg}$ (74 pacientes).

Quanto ao número de valvas, $121(62,4 \%)$ pacientes tiveram 1 valva abordada, 55 (28,3\%) 2 valvas e $18(9,3 \%) 3$ valvas. Segundo o tipo de procedimento, $16(8,2 \%)$ pacientes foram submetidos a operações conservadoras, 144 (74,2\%) a implante de biopróteses e $34(17,5 \%)$ a implante de próteses mecânicas.

Quanto a posição, esta foi mitral em $73(37,6 \%)$ pacientes; aórtica em 45 (23,2\%); mitral e aórtica em 32 (16,5\%); mitral e tricúspide em 23 (11,8\%); mitral, aórtica e tricúspide em 18 (9,3\%); tricúspide em $2(1,0 \%)$ e aórtica e tricúspide em $1(0,5 \%)$. Segundo as operações associadas, 7 pacientes $(3,6 \%)$ foram submetidos a tratamento cirúrgico de abscesso de anel e $3(1,5 \%)$ a revascularização do miocárdio.

\section{Análise Estatística dos Dados}

Para avaliar os fatores preditivos da ocorrência de óbito, foi verificada a associação das variáveis pré e intra-operatórias com o óbito, através de análise univariada com o teste Qui- quadrado de Pearson ou o teste exato de Fisher, para variáveis com baixa frequiência esperada.

Posteriormente, as variáveis selecionadas nesta análise foram utilizadas no ajuste de modelos de regressão logística ${ }^{(7)}$ para avaliar quais variáveis estão mais fortemente associadas à probabilidade de óbito. O nível de significância estabelecido para análise foi de 5\% e todos os cálculos foram realizados por meio do sistema SAS (Statistical Analysis System) ${ }^{(8)}$.

O óbito foi considerado como tendo ocorrido em qualquer dia do pós-operatório durante a hospitalização ${ }^{(9)}$.

\section{RESULTADOS}

A mortalidade hospitalar foi de 8,8\% (17 pacientes). Aprincipal causa de mortalidade hospitalar foi o baixo débito cardíaco, em 6 $(35,3 \%)$ pacientes. Outras causas foram: insuficiência de múltiplos órgãos em 5 (29,4\%) pacientes, sepse em 3 (17,6\%), síndrome da angústia respiratória do adulto (SARA) em 1 (5,9\%), acidente vascular cerebral em $1(5,9 \%)$ e distúrbio de coagulação em 1 $(5,9 \%)$ paciente.

\section{Análise Univariada}

As variáveis $\mathrm{CF}$, FEVE, nível sérico de creatinina, AP, tempo de CEC, tempo de pinçamento aórtico, operações associadas e volume de sangramento foram preditivas de mortalidade hospitalar(Tabela 1).

Tabela 1. Análise Univariada: variáveis significativas

\begin{tabular}{lcccc}
\hline VARIÁVEL & PCTS & MORTALIDADE & $\%$ & p \\
\hline CF & 20 & & & \\
II & 104 & 4 & 3,8 & \\
III & 70 & 13 & 18,6 & \\
IV & & & & \\
FEVE & 55 & 10 & 18,2 & 0,003 \\
$<=65 \%$ & 139 & 7 & 5,0 & \\
$>65 \%$ & & & & \\
Creatinina & 179 & 12 & 6,7 & 0,005 \\
$<=1,5 \mathrm{mg} / \mathrm{dl}$ & 15 & 5 & 33,3 & \\
$>1,5 \mathrm{mg} / \mathrm{dl}$ & & & & \\
AP & 32 & 6 & 18,7 & 0,028 \\
$<=60 \%$ & 162 & 11 & 6,8 & \\
$>60 \%$ & & & & \\
Tempo de CEC & 134 & 6 & 4,5 & 0,002 \\
$<=120 \mathrm{~min}$ & 60 & 11 & 18,3 & \\
$>120 \mathrm{~min}$ & & & & \\
Tempo de Pinçamento Aórtico & 115 & 6 & 5,2 & 0,035 \\
$<=90$ min & 79 & 11 & 13,9 & \\
$>$ > 90 min & & & & \\
Operações Associadas & 184 & 14 & 7,6 & 0,004 \\
Nenhuma & 7 & 3 & 42,8 & \\
Abscesso de Anel Valvar & 3 & & & \\
Revascularização do Miocárdio & 3 & & & \\
Volume de Sangramento & 120 & 6 & 5,0 & 0,018 \\
$<=8,6 \mathrm{ml} / \mathrm{kg}$ & 74 & 11 & 14,9 & \\
$>>8,6$ ml/kg & & & & \\
\hline
\end{tabular}

PCTS: pacientes; CF- classe funcional, FEVE - fração de ejeção do ventrículo esquerdo; AP - atividade de protrombina; CECcirculação extracorpórea. 
O sexo não influenciou a mortalidade significativamente, embora tenha sido maior no sexo feminino $(10,5 \%)$ do que no masculino $(4,7 \%)$. A idade também não foi preditiva de mortalidade, sendo esta ausente no pacientes com menos de 16 anos, de $9,0 \%$ nos pacientes entre 17 e 65 anos e de $11,4 \%$ para os acima de 65 anos. O número de operações prévias também não interferiu de forma significativa, embora esta tenha sido maior na primeira reoperação $(10,3 \%)$ do que na segunda $(5,7 \%)$ ou terceira $(6,7 \%)$. Não houve mortalidade nas quartas ou quintas reoperações. Os pacientes com operações conservadoras prévias tiveram mortalidade de $2,3 \%$ contra $10,6 \%$ nos pacientes com substituições valvares prévias. $\mathrm{O}$ ritmo cardíaco e o caráter da operação também não foram preditivos de mortalidade hospitalar.

As variáveis ecoDopplecardiográficas DDVE, DSVE e PSVD e laboratoriais rTTPA e contagem de plaquetas não influenciaram de forma significativa a mortalidade hospitalar.

O número de valvas não foi preditivo de mortalidade, embora esta tenha sido menor nos pacientes com 1 valva $(5,8 \%)$ do que nos com $2(14,5 \%)$ ou 3 valvas $(11,1 \%)$. O tipo de procedimento também não um fator de risco, sendo que a mortalidade ausente para os procedimentos conservadores, 2,9\% para o implante de próteses mecânicas e $11,1 \%$ para o implante de biopróteses. Quanto a posição, a mortalidade foi de 2,7\% para a mitral, $8,9 \%$ para a aórtica, $9,4 \%$ para a mitroaórtica, $21,7 \%$ para a mitro-tricuspídea, $11,1 \%$ para a mitroaórtica-tricuspídea e 33,3\% para as demais associações.

\section{Análise Multivariada - Regressão Logística}

Considerando-se as variáveis pré e intra-operatórias que apresentaram significância na análise univariada, foi ajustada uma regressão logística para avaliar a importância destas variáveis na ocorrência de óbito hospitalar. Na Tabela 2 temos os resultados do ajuste.

Tabela 2. Regressão Logística: variáveis independentes.

\begin{tabular}{lccccc}
\hline EFEITO & ESTIMATIVA & $\begin{array}{c}\text { ERRO } \\
\text { PADRÃO }\end{array}$ & p & $\begin{array}{c}\text { ODDS- } \\
\text { RATIO }\end{array}$ & I.C. 95\% \\
\hline Creatinina $>1,5 \mathrm{mg} / \mathrm{dl}$ & 1,8057 & 0,7024 & 0,010 & 6,084 & $(1,536 ; 24,105)$ \\
CF IV & 1,7030 & 0,6185 & 0,006 & 5,490 & $(1,634 ; 18,454)$ \\
Tempo de CEC $>120$ min & 1,3935 & 0,5725 & 0,015 & 4,029 & $(1,312 ; 12,374)$ \\
Intercepto & $-4,1865$ & 0,6318 & $<0,001$ & & \\
\hline
\end{tabular}

CF- classe funcional; CEC - circulação extracorpórea.

\section{COMENTÁRIOS}

O número de reoperações valvares tem aumentado nos últimos anos devido a vários fatores, como: maior sobrevida após as operações valvares, crescente expectativa de vida da população e a ampla utilização de biopróteses na nossa população, que devido à sua vida útil limitada pela disfunção estrutural requerem novas intervenções cirúrgicas. Na nossa casuística, $66,5 \%$ dos pacientes apresentavam rotura ou calcificação de bioprótese.
A mortalidade hospitalar nas reoperações valvares, em geral, é considerada maior do que a das primeiras operações. Nesta série, a mortalidade global foi de $8,8 \%$, sendo de $2,7 \%$ para a posição mitral e $8,9 \%$ para a posição aórtica. ANTUNES \& MAGALHÃES ${ }^{(10)}$ descrevem $12 \%$ de mortalidade para reoperações de prótese mitral e ANTUNES ${ }^{(11)}$ descreve $9 \%$ para a aórtica. JAMIESON et al. ${ }^{(12)}$, em estudo multicêntrico do Database Committee of the Society of Thoracic Surgeons, analisaram 86.580 pacientes submetidos à operação valvar entre 1986 e 1995, com taxas de mortalidade de 5,9\% para reoperações aórticas eletivas e 6,2\% para reoperações mitrais eletivas. No nosso meio, BARROZO et al. ${ }^{(13)}$ descrevem $9,89 \%$ de mortalidade hospitalar em 697 pacientes submetidos a reoperações valvares.

A CF pré-operatória é, sem dúvida, um importante preditor de mortalidade hospitalar. Vários autores ${ }^{(14,15,16)}$ identificaram a CF IV como fator preditivo independente de mortalidade. Segundo HUSEBYE et al. ${ }^{(17)}$, em análise de 552 reoperações valvares realizadas na Mayo Clinic, a mortalidade hospitalar foi significativamente maior na CF IV, recomendando que quando uma disfunção protética significativa for diagnosticada deve-se indicar a intervenção cirúrgica mesmo em pacientes oligossintomáticos, a fim de minimizar o risco operatório e melhorar a sobrevida tardia.

AFEVE foi um fator de risco independente para a mortalidade hospitalar. BORTOLOTTI et al. ${ }^{(1)}$ demonstraram que os pacientes sobreviventes no pós-operatório imediato tinham FEVE significativamente maior. TURINA e TURINA ${ }^{(18)}$ não mostraram diferença estatisticamente significativa entre pacientes com FEVE menor e maior que 50\%.

A creatinina sérica também influenciou significativamente a mortalidade hospitalar. BIGLIOLI et al. ${ }^{(4)}$, em análise univariada de variáveis contínuas, identificaram o nível sérico de creatinina como preditivo para mortalidade hospitalar, em concordância com os nossos resultados. AKINS et al. ${ }^{(19)}$ demonstraram a influência da insuficiência renal pré-operatória na morbidade e na mortalidade hospitalar, sendo esta um fator preditivo independente na análise multivariada, como também apresentrado por PIEHLER et al. ${ }^{(14)}$.

A AP também foi um fator preditivo para mortalidade hospitalar na análise univariada. A alteração da AP indica depleção de fatores vitamina $\mathrm{K}$ dependentes, principalmente com o fator $\mathrm{V}$ em níveis normais. Não existem na literatura relatos correlacionando a AP com a mortalidade hospitalar.

Quanto ao tempo de CEC, este influenciou significativamente a mortalidade hospitalar na análise multivariada. Este fator de risco também foi descrito por vários autores, como BIGLIOLI et al. ${ }^{(4)}$ e CAUS et al. ${ }^{(20)}$. O tempo de CEC longo está relacionado com outros fatores, como operações prolongadas, multivalvares, reconstrução de anel na endocardite com abscesso anular, ou pacientes com disfunção ventricular, que necessitam de assistência circulatória por um tempo mais longo. O tempo de pinçamento aórtico também foi preditivo de mortalidade hospitalar, corroborando com vários trabalhos da 
literatura, como os de BIGLIOLI et al. ${ }^{(4)}$ e PANSISI et al. ${ }^{(16)}$. Esta é uma variável que também está diretamente relacionada com a complexidade técnica da operação.

A presença de operações associadas também foi preditiva de mortalidade hospitalar na análise univariada, como nos trabalhos de BORTOLOTTI et al. ${ }^{(1)}$, PIEHLER et al. ${ }^{(14)} \mathrm{e}$ RIZZOLI et al. ${ }^{(15)}$. Na nossa série, a associação com o tratamento cirúrgico do abscesso de anel valvar foi a responsável por este resultado. $\mathrm{O}$ abscesso de anel valvar é uma afecção associada com alta morbidade e mortalidade hospitalares, como demonstrado por LYTLE et al. ${ }^{\left({ }^{(2)}\right)}$ e POMERANTZEFF et al. ${ }^{(22)}$.

$O$ volume de sangramento intra-operatório também foi um fator preditivo de mortalidade na análise univariada. Esta variável não foi descrita na literatura como preditiva de mortalidade hospitalar e não é uma variável independente.

Com relação ao sexo, nossos resultados foram semelhantes aos descritos por COHN et al. ${ }^{(23)}$ e ANTUNES ${ }^{(24)}$ que não encontraram influência do sexo na mortalidade hospitalar. A idade também não foi um fator preditivo de mortalidade. Alguns autores, como PIEHLER et al. ${ }^{(14)}$, identificaram a idade como um fator independente de mortalidade hospitalar em reoperações valvares.

Quanto ao número de operações prévias, apesar da mortalidade ter sido superior na primeira do que na segunda ou terceira reoperação, esta não foi estatisticamente significativa. Na série apresentada PIEHLER et al. ${ }^{(14)}$, tanto o número de operações cardíacas prévias quanto o número de operações valvares prévias foram preditivos de mortalidade na análise multivariada.

Os pacientes com operações prévias conservadoras apresentaram uma mortalidade hospitalar de $2,3 \%$, enquanto os submetidos a substituições valvares prévias apresentaram mortalidade de $10,6 \%$, porém esta diferença não foi estatisticamente significativa. Resultado semelhante foi apresentado por LYTLE et al. ${ }^{(3)}$, o que reforça a indicação de procedimentos valvares conservadores sempre que possível.

A fibrilação atrial pode ser um fator de risco para a mortalidade hospitalar, pois pode provocar baixo débito cardíaco no pósoperatório ou predispor a acidentes tromboembólicos. $\mathrm{Na}$ literatura, a fibrilação atrial é identificada como um fator de risco em operações valvares ${ }^{(25)}$. Na nossa série, bem como na de ANDRADE et al. ${ }^{(26)}$, a fibrilação atrial não influenciou de forma estatisticamente significativa a mortalidade hospitalar.

Vários autores como BORTOLOTTI et al. ${ }^{(1)}$, BIGLIOTI et al. ${ }^{(4)}$, RIZZOLI et al. ${ }^{(15)}$, PANSISI et al. ${ }^{(16)}$, HUSEBYE et al. ${ }^{(17)}$ e AKINS et al. ${ }^{(19)}$ identificaram o caráter de emergência nas reoperações valvares como um fator preditivo independente de mortalidade. Na nossa série, a mortalidade foi ausente nas reoperações de emergência, porém tratam-se apenas de 5 casos.

Os DDVE e DSVE não influenciaram a mortalidade hospitalar, assim como na série apresentada por BIGLIOLI et al. ${ }^{(4)}$. Segundo CARABELLO et al. ${ }^{(27)}$, o único fator preditivo independente de má evolução após operação valvar é o índice de volume sistólico. A mortalidade hospitalar também não sofreu influência significativa da PSVD. Na análise multivariada apresentada por BIGLIOLI et al. ${ }^{(4)}$, o valor da pressão da artéria pulmonar não influenciou significativamente a mortalidade.
Resultado similar foi obtido por AKINS et al. ${ }^{(19)}$, que analisaram a pressão sistólica da artéria pulmonar.

Outras alterações nos exames de coagulação, como a rTTPA e a contagem de plaquetas não foram identificadas como fatores de risco para a mortalidade hospitalar, embora esta tenha sido maior nos pacientes com essas alterações.

O número de valvas abordadas também não foi preditivo de mortalidade hospitalar. Esta variável foi identificada como fator de risco para mortalidade em outras séries da literatura $(14,16)$. A posição também não foi um fator preditivo de mortalidade, embora esta tenha sido menor na posição mitral. ROSSITER et al. ${ }^{(28)}$ sugerem que a disparidade entre as posições aórtica e mitral pode ser explicada pela maior ocorrência de endocardite em prótese na posição aórtica. Por sua vez, LYTLE et al. ${ }^{(3)}$ associaram estes resultados com a maior associação da doença arterial coronariana na posição aórtica. O tipo de procedimento realizado não influenciou de forma significativa a mortalidade, com $11,1 \%$ para a substituição por biopróteses, $2,9 \%$ para a substituição por próteses mecânicas e ausente para os procedimentos conservadores.

COHN et al. ${ }^{(23)}$ demonstraram a importância do aprimoramento da técnica para os resultados nas reoperações valvares. Segundo estes autores, algumas alterações na técnica operatória contribuíram para a melhora dos resultados, como a utilização mais freqüente da circulação extracorpórea via fêmorofemoral, a hipotermia sistêmica, a cardioplegia sangüínea, a monitorização no pós-operatório das pressões de artéria e capilar pulmonares e da pressão venosa central, o uso de marcapasso provisório atrial e ventricular e o uso da autotransfusão automática intra-operatória.

Outro aspecto que ganha importância na literatura é a indicação precoce em pacientes oligossintomáticos ou assintomáticos ${ }^{(29,30)}$ com disfunção estrutural de prótese, prevenindo que estes sejam operados em classe funcional avançada ou em condições de emergência.

Em suma, o aprimoramento das técnicas, a análise individual de cada caso, com especial atenção para os fatores de risco, de forma a preparar melhor os pacientes no período pré-operatório ou indicar a operação em melhores condições clínicas, são de importância fundamental para a obtenção de bons resultados nas reoperações valvares.

\section{CONCLUSÕES}

-ACFIV, o nível sérico de creatinina $>1,5 \mathrm{mg} / \mathrm{dl}$, a FEVE <= $65 \%$, a AP $<=60 \%$, o tempo de CEC $>120$ minutos, o tempo de pinçamento aórtico $>90$ minutos, as operações associadas e o volume de sangramento $>8,6 \mathrm{ml} / \mathrm{Kg}$ foram fatores de risco preditivos para mortalidade hospitalar na análise univariada.

-ACF IV, o nível sérico de creatinina $>1,5 \mathrm{mg} / \mathrm{dl}$ e o tempo de $\mathrm{CEC}>120$ minutos foram identificados como fatores de risco preditivos independentes de mortalidade hospitalar na análise multivariada. 


\section{REFERÊNCIAS BIBLIOGRÁFICAS}

1. Bortolotti U, Milano A, Mossuto E, Mazzaro E, Thiene G, Casarotto D-Early and late outcome after reoperation for prosthetic valve dysfunction: analysis of 549 patients during a 26- year period. J Heart Valve Dis 1994; 3: 81-7.

2. Sener E, Yamak B, Katircioglu SF et al. - Risk factors of reoperations for prosthetic heart valve dysfunction in the ten years 1984- 1993. Thorac Cardiovasc Surg 1995; 43: 148-52.

3. Lytle BW, Cosgrove DM, Taylor PC et al. - Reoperations for valve surgery: perioperative mortality and determinants of risk for 1000 patients, 1958- 1984. Ann Thorac Surg 1986; 42: 632-43.

4. Biglioli PDI, Matteo S, Parolari A, Antona C, Arena V, Sala A Reoperative cardiac valve surgery: a multivariable analysis of risk factors. Cardiovasc Surg 1994; .2: 216-22.

5. Pomerantzeff PMA, Yochitomi Y, Fabri HA et al. - Reoperações valvares: experiência do InCor- FMUSP. Rev Bras Cir Cardiovasc 1991; 6: 182-9.

6. Bueno RM, Neto VA, Melo RFA - Fatores de risco em operações valvares: análise de 412 casos. Rev Bras Cir Cardiovasc 1997; 12: 348-58.

7. Hosmer Jr DW, Lemeshow S - Applied logistic regression. John Wiley \& Sons, 1976: 75-118.

8. SAS Institute Inc., SAS/ STAT? User's Guide. Version 6. Fourth Edition. Volumes 1,2. Cary, NC: SAS Institute Inc., 1989.

9. Edmunds Jr LH, Clark RE, Cohn LH, Grunkemeier GL, Miller DC, Weisel RD - Guidelines for reporting morbidity and mortality after cardiac valvular operations. J Thorac Cardiovasc Surg 1996; 112: 708-11.

10. Antunes MJ, Magalhães MP - Isolated replacement of a prosthesis or a bioprosthesis in the mitral valve position. Am J Cardiol 1987; 59: $346-9$

11. Antunes MJ - Isolated replacement of a prosthesis or a bioprosthesis in the aortic valve position. Am J Cardiol 1987; 59: 350-2.

12. Jamieson WR, Edwards FH, Schwartz M, Bero JW, Clark RE, Grover FL - Risk stratification for cardiac valve replacement. National Cardiac Surgery Database. Ann Thorac Surg 1999; 67: 943-51.

13. Barrozo CAM, Santos RC, Sgarbi CJ - Reoperação em valvopatias: análise de 697 pacientes. Rev Bras Cir Cardiovasc 1994, 9: 10912.

14. Piehler JM, Blackstone EH, Bailey KR et al. - Reoperation on prosthetic heart valves: patient- specific estimates of in- hospital events. J Thorac Cardiovasc Surg 1995; 109: 30-48.

15. Rizzoli G, Bottio TDE, Perini 1, Scalia D, Thiene G, Casarotto D Multivariate analysis of survival after malfunctioning biological and mechanical prosthesis replacement. Ann Thorac Surg 1998; 66: S88-94.

16. Pansisi S, Ottino G, Forsennati PG et al. Reoperations on heart valve prostheses: an analysis of operative risks and late results. Ann Thorac Surg 1990; 50: 590-6.

17. Husebye DG, Pluth JR, Piehler JM et al. - Reoperation on prosthetic heart valves: an analysis of risk factors in 552 patients. $J$ Thorac Cardiovasc Surg 1983; 86: 543-52.

18. Turina J, Turina $\mathrm{M}$ - Left ventricular function and valvular reoperations. J Heart Valve Dis 1995; 4(Supl II): S223-9.

19. Akins CW, Buckley MJ, Daggett WM et al. - Risk of reoperative valve replacement for failed mitral and aortic bioprostheses. Ann Thorac Surg 1998; 65:1545-52.

20. Caus T, Mesana T, Mouly A, Guez P, Tapia M, Montiès JR Remplacements valvulaires itératifs; prognostic et résultats. Arch Mal Coeur Vaiss 1995; 88: 35-41.

21. Lytle BW, Priest BP, Taylor PC etal. - Surgical treatment of prosthetic valve endocarditis. J Thorac Cardiovasc Surg 1996; 111: 198-210.

22. Pomerantzeff PMA, Brandão CMA, Mansur AJ et al. - Tratamento cirúrgico do abcesso de anel valvar associado a endocardite bacteriana: resultados imediatos e tardios. Rev Bras Cir Cardiovasc 1996; 11: 259-62.

23. Cohn LH, Aranki SF, Rizzo RJ et al. - Decrease in operative risk of reoperative valve surgery. Ann Thorac Surg 1993; 56: 15-21.

24. Antunes MJ - Reoperations on cardiac valves. J Heart Valve Dis 1992; 1: 15-28.

25. Cosgrove DM. - Evaluation of perioperative risk factors. J Card Surg 1990; 5(3): 227-30.

26. Andrade IG, Cartier R, Panisi P, Ennabli K, Grondin CM - Factors influencing early and late survival in patients with combined mitral valve replacement and myocardial revascularization and in those with isolated replacement. Ann Thorac Surg 1987;44: 607-13.

27. Carabello BA, Williams H, Gash AK et al. - Hemodynamic predictors of outcome in patients undergoing valve replacement. Circulation 1986; 74: 1309-16.

28. Rossiter SJ, Miller DC, Stinson EB, Oyer PE, Reitz BA, Shumway NE - Aortic and mitral prosthetic valve reoperations: early and late results. Arch Surg 1979; 114: 1279-83.

29. Blackstone EH, Kirklin JW. - Recommendations for prophylactic removal of heart valve prostheses. J Heart Valve Dis 1992; 1: 3-14.

30. Bortolotti U, Milano A, Guerra F et al. - Failure of Hancock pericardial xenografts: is prophylactic bioprosthetic replacement justified? Ann Thorac Surg 1991; 51: 430-7. 